\title{
Electric-field-induced coherent coupling of the exciton states in a single quantum dot
}

\author{
A. J. Bennett ${ }^{1 \star}$, M. A. Pooley ${ }^{1,2}$, R. M. Stevenson ${ }^{1}$, M. B. Ward ${ }^{1}$, R. B. Patel ${ }^{1,2}$, A. Boyer de la Giroday ${ }^{1,2}$, \\ N. Sköld' ${ }^{1}$ I. Farrer ${ }^{2}$, C. A. Nicoll ${ }^{2}$, D. A. Ritchie ${ }^{2}$ and A. J. Shields ${ }^{1}$
}

The signature of coherent coupling between two quantum states is an anticrossing in their energies as one is swept through the other. In single semiconductor quantum dots containing an electron-hole pair the eigenstates form a twolevel system that can be used to demonstrate quantum effects in the solid state, but in all previous work these states were independent $^{1-5}$. Here we describe a technique to control the energetic splitting of these states using a vertical electric field, facilitating the observation of coherent coupling between them. Near the minimum splitting the eigenstates rotate in the plane of the sample, being orientated at $45^{\circ}$ when the splitting is smallest. Using this system we show direct control over the exciton states in one quantum dot, leading to the generation of entangled photon pairs.

It is well known that the exchange interaction in single semiconductor dots results in the exciton eigenstates being linearly polarized with an energy difference known as the fine-structure splitting $^{6,7}$ (FSS, $\left.|s|\right)$. The magnitude of the FSS is determined by anisotropy in the strain, shape and composition of the dot, in addition to a contribution from the crystal inversion asymmetry ${ }^{8}$. When the FSS is smaller than the linewidth, the biexciton-toexciton-to-empty cascade can lead to the emission of polarizationentangled photon pairs ${ }^{9,10}$, which has motivated much work on this subject.

Recently, theoretical work has suggested that for realistic straintuned dots a minimum in the FSS $\left(s_{0}\right)$ will be observed of the order of $3 \mu \mathrm{eV}$, owing to the symmetry of the crystal ${ }^{11}$, but this has yet to be confirmed by experiment. Some success at tuning the FSS has been reported using strain ${ }^{12}$ but this did not reach zero. Other tuning techniques such as magnetic field ${ }^{1,6}$, strong coherent lasers $^{2,3}$, lateral electric field $\mathrm{d}^{4,5,13}$ and vertical electric field ${ }^{14,15}$ have been investigated but those that have been able to minimize the FSS have seen the states cross ${ }^{1-5}$.

Arguably, tuning the FSS with vertical electric field is the most practical technique yet reported, but the low confinement energies have limited the fields that can be applied to a few tens of kilovolts per centimetre before carriers tunnel from the dot, so relatively small changes in FSS were observed ${ }^{14,15}$. Here we demonstrate a design of heterostructure (Fig. 1a) that allows much larger electric fields to be applied. Then both eigenstates of the exciton Stark shift at different rates, leading to a linear change in the FSS of over $100 \mu \mathrm{eV}$. At appropriate fields we observed for the first time anticrossings in the energies of the two exciton levels. Near the anticrossing, hybridization of the polarization states of the upper and lower branches leads to their rotation in the plane of the sample. In a dot that has a small anticrossing (below the homogeneous linewidths), we are thus able to demonstrate
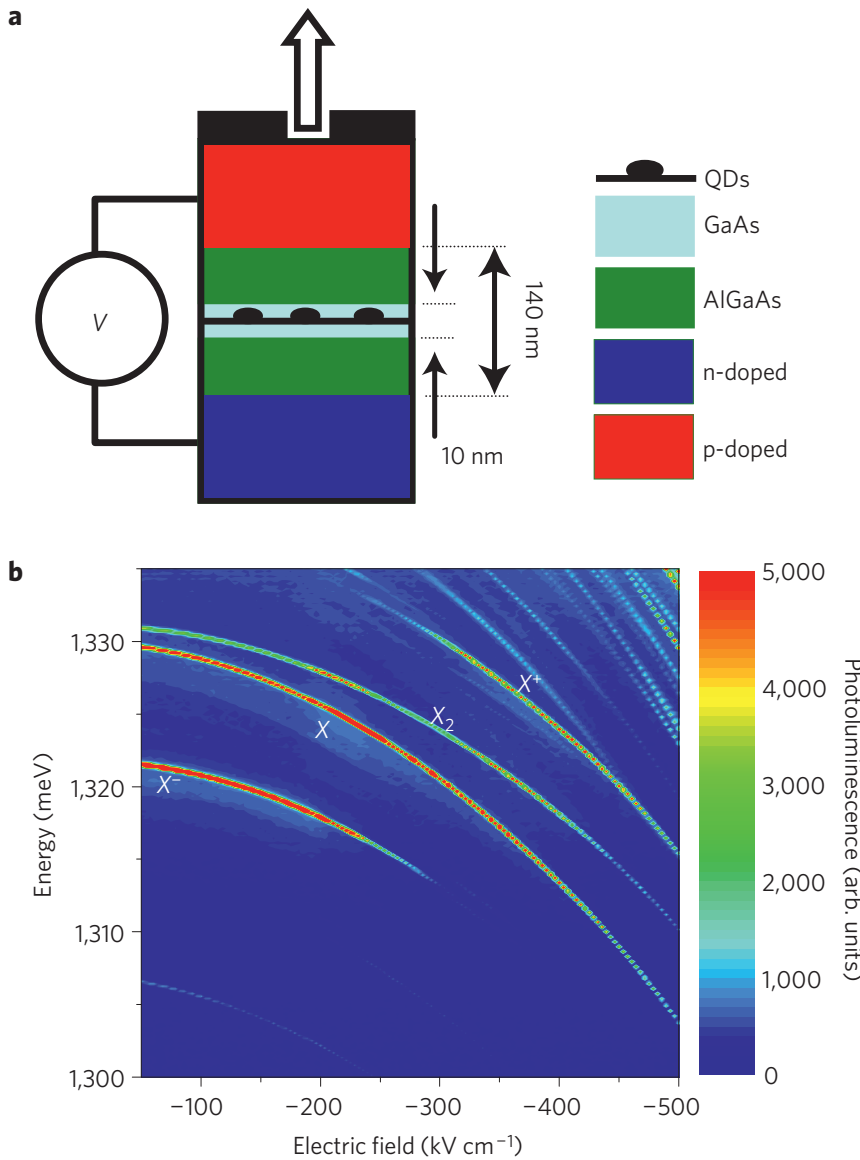

Figure 1 | Device design and observed giant Stark shift of the excitonic transitions. a, Heterostructure design. b. Typical plot of photoluminescence versus electric field for a single quantum dot.

control of entangled-photon-pair emission using electric field. Other dots exhibit a large minimum splitting (FSS greater than the homogeneous linewidths) and a coherent superposition of the usual exciton states is observed.

As electric field is applied all transitions Stark shift with the applied field (Fig. 1b), following the form $E=E_{0}-p F+\beta F^{2}$, where $p$ is the permanent dipole moment in the $z$ direction, $\beta$ is the polarizability and $F$ is the applied field ${ }^{13,16,17}$. At large values of FSS, the exciton eigenstates can be thought of as radiating dipoles aligned along the [110] and [110] crystal axes

${ }^{1}$ Toshiba Research Europe Limited, Cambridge Research Laboratory, 208 Science Park, Milton Road, Cambridge CB4 0GZ, UK, ${ }^{2}$ Cavendish Laboratory, Cambridge University, J. J. Thomson Avenue, Cambridge CB3 OHE, UK. *e-mail: anthony.bennett@crl.toshiba.co.uk. 


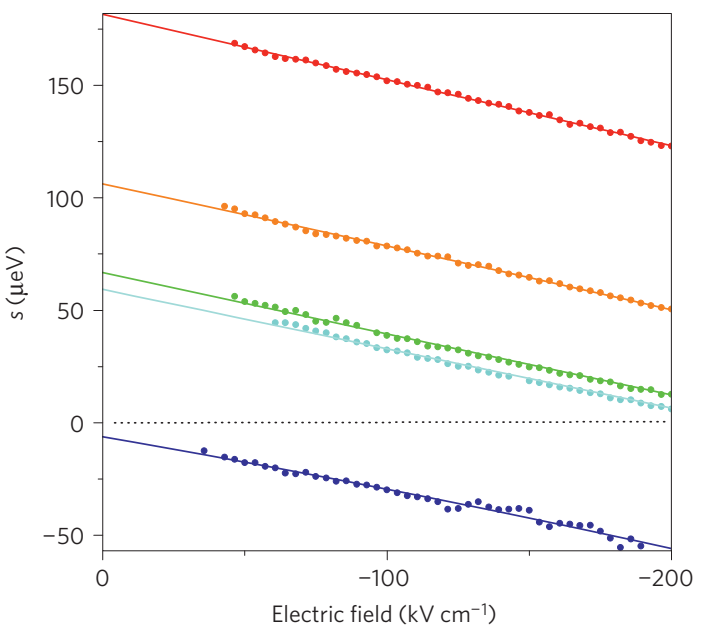

Figure 2 | Fine-structure splitting $s$ as a function of electric field for five dots with naturally different $s$ at low field. $s$ varies in the same manner for all dots in this sample. One dot has an inverted fine-structure at all fields, with the lowest-energy exciton state orthogonal to other dots we have studied, and is plotted with a negative $s$.

(see Supplementary Information). This orientation is mapped onto the emitted photon's polarization $\left(X_{\mathrm{H} / \mathrm{V}}\right.$ denoting photons of horizontal or vertical polarization). Remarkably, away from the minimum FSS all neutral states exhibit a linear change in the magnitude of the FSS with electric field at a rate $\gamma=-0.285 \pm$ $0.019 \mu \mathrm{eV} \mathrm{kV}^{-1} \mathrm{~cm}$ (Fig. 2). Even the unusual dot we have identified that has its lowest-energy exciton eigenstate orthogonal to all others in this sample, and thus is plotted on Fig. 2 with a negative $s$, has the same gradient. This value of $\gamma$ is independent of the FSS at zero field, emission energy, binding energy and the Stark shift parameters $p$ and $\beta$. The observed linear shift may be explained by the fact that the two eigenstates of the neutral exciton have slightly different confinement potentials in the two directions. This leads to different permanent dipole moments along the $z$ direction ${ }^{17}$ giving $p_{\mathrm{H}}-p_{\mathrm{V}}=\gamma$. However, the polarizability of these states is unaffected by this in-plane anisotropy ${ }^{16}$ and is controlled only by the height of the confinement potential, which is the same for both $X_{\mathrm{H}}$ and $X_{\mathrm{V}}$. Thus, the measured value of $\gamma$ means the dipole moments of the two exciton eigenstates must differ by a few per cent. The fact that $\gamma$ is so similar in our ensemble suggests that the dots all have comparable in-plane anisotropy, and that this value could be manipulated by changing the shape of the dots.

This ability to continuously tune the FSS over such a large range allows us to observe an anticrossing in the neutral excitonic levels. The variation of the FSS with field is shown in Fig. $3 \mathrm{c}$ for three dots, clearly indicating coherent coupling between these states. The splitting is well described by a simple model:

$$
E\left(\begin{array}{c}
\cos \theta \\
\sin \theta
\end{array}\right)=\left(\begin{array}{cc}
E_{0} & s_{0} / 2 \\
s_{0} / 2 & E_{0}-\gamma\left(F-F_{0}\right)
\end{array}\right)\left(\begin{array}{c}
\cos \theta \\
\sin \theta
\end{array}\right)
$$

where $s_{0} / 2$ quantifies the coupling between the states, $F$ is electric field, $F_{0}$ is the field at minimal splitting and the energy levels move together with a rate $\gamma$ in the absence of the coupling term $s_{0} / 2$. This is the same form of equation that is used to describe coupled harmonic oscillators ${ }^{18}$, strong light-matter coupling ${ }^{19}$ and anticrossings in the states of molecular systems ${ }^{20}$. When $\gamma\left(F-F_{0}\right) \ggg s_{0}$, the natural basis to choose is that aligned with the crystal axes, and nearer $\left(F-F_{0}\right)=0$ the eigenstates will be a coherent mixture, with components $\sin \theta$ and $\cos \theta$. In this system the parameter $\theta$ is a real angle that describes the orientation of the

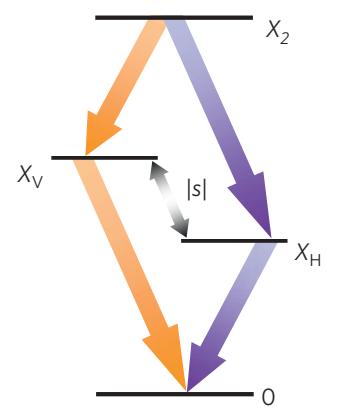

b

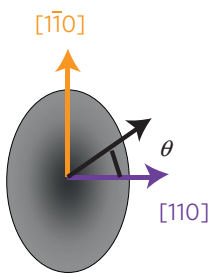

C

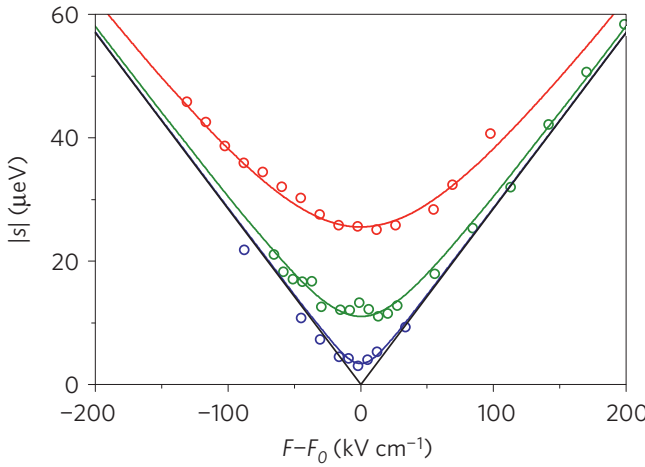

d

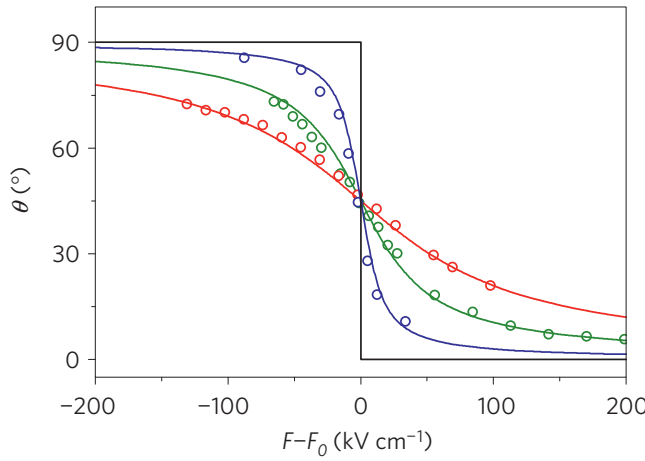

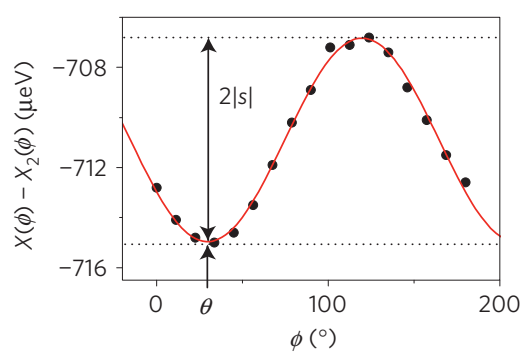

f

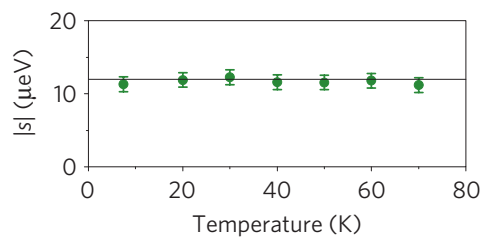

$\mathbf{g}$

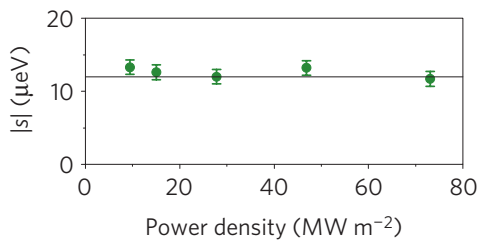

Figure 3 | Characteristics of electric-field-induced coherent coupling of exciton states in a single quantum dot. a, Energy levels in the neutral cascade of a single dot. $\mathbf{b}$, Orientation of the eigenstates relative to the crystal axes. $\mathbf{c}, \mathbf{d},|s|$ and $\theta$ as a function of the electric field (offset by $F_{0}$ ) for three quantum dots with values of $s_{0}$ equal to $25.5,12.0$ and $3.0 \mu \mathrm{eV}$ and $F_{0}$ of $-140.5,-234.5$ and $-98.0 \mathrm{kV} \mathrm{cm}^{-1}$. The black line shows what would be expected from a dot with vanishing magnitude of anticrossing. $\mathbf{e}$, The result of a typical measurement of the $\left[X(\phi)-X_{2}(\phi)\right]$ from which we can extract $|s|$ and $\theta$.

$\mathbf{f}, \mathbf{g}$, Measurements of the amplitude of the anticrossing for one of the dots as a function of temperature (f) and excitation intensity (g). 
a

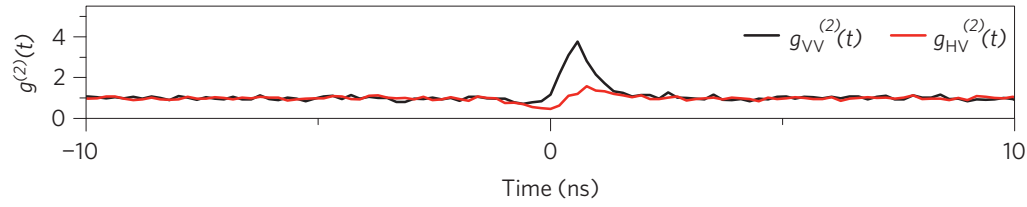

b

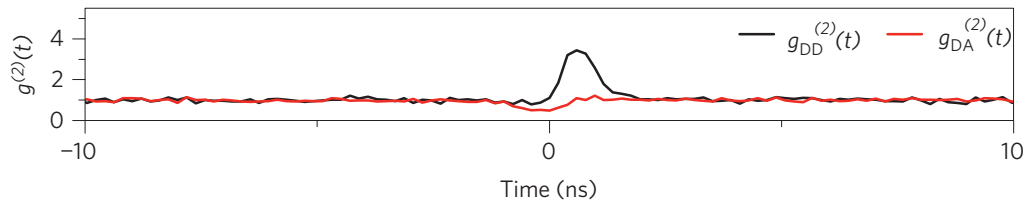

c
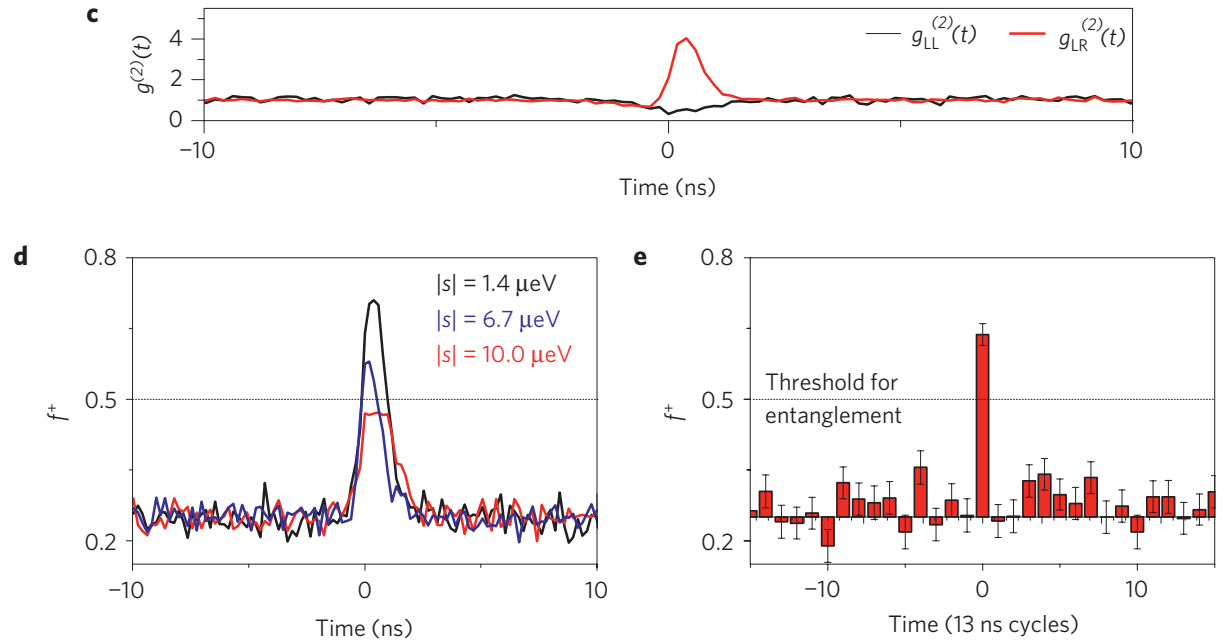

Figure 4 | Entanglement in the cascade emission of a dot with $s_{0}=1.5 \mu \mathrm{eV}$. a-c, Polarized cross-correlations between $X$ and $X_{2}$ photons in three orthogonal polarization bases: rectilinear $\{H, V\}$ (a), diagonal $\{D, A\}(\mathbf{b})$ and circular $\{L, R\}$ (c). d, Fidelity of the emitted state, for three different values of $|s|$. e, Fidelity of the emission with pulsed optical excitation, considering only those $X$ photons emitted within 100 ps of a preceding biexciton photon at $s=1.5 \mu \mathrm{eV}$.

eigenstates relative to the crystal lattice (Fig. 3b). The eigenvalues, $E_{ \pm}$, and eigenvectors are well known, with

$$
\begin{gathered}
E_{ \pm}=E_{0}-\frac{\gamma\left(F-F_{0}\right)}{2} \pm \frac{1}{2} \sqrt{\gamma^{2}\left(F-F_{0}\right)^{2}+s_{0}^{2}} \\
\theta= \pm \tan ^{-1}\left[\frac{s_{0}}{\gamma\left(F-F_{0}\right) \pm\left(E_{-}-E_{+}\right)}\right]
\end{gathered}
$$

We note that this simple model has degenerate solutions where the eigenstates rotate either clockwise or anticlockwise when they approach $\left(F-F_{0}\right)=0$. However, in practice we observe that each dot has a clear preference to rotate one way or another in the plane of the sample, but never into the circular basis. Our measurements are consistent with the ensemble having no preferred direction of rotation. The origin of this handedness in individual dots is unknown, but may be determined by local defects or fields in the semiconductor.

Experimental data for three dots with varying sizes of anticrossing and $F_{0}$ are shown in Fig. $3 \mathrm{c}$,d, where all three dots rotate in the same direction. These data show excellent agreement between the simple model and the experiment, indicative of a coherent coupling that can be activated with electric field.

A study of 22 dots revealed values of $s_{0}$ in the range $0.7-42.9 \mu \mathrm{eV}$, with lower values being observed more frequently (see Supplementary Information). No trend was observed between the magnitude of this value and other parameters associated with the electronic states of the dot, such as the FSS at $F=0, F_{0}$, the emission energy or $\left(X(\phi)-X_{2}(\phi)\right)$.
Coupled pairs of oscillators have two normal mode frequencies that depend in part on the damping, or decoherence, affecting those individual oscillators ${ }^{18}$. For this reason we have investigated varying the sample temperature (from 4 to $70 \mathrm{~K}$, Fig. 3f) and the excitation power, changing the ratio of exciton to biexciton intensity from 10:1 to $1.3: 1$ (Fig. 3g) for the dot with $\left|s_{0}\right|=12.0 \mu \mathrm{eV}$. Both of these factors will vary the decoherence experienced by a single $\operatorname{state}^{21}$, but have no effect on the magnitude of the coupling. This suggests that although these external factors may cause decoherence of the individual states (such as the $T_{2}$ time) it does not affect the timescale on which the two states dephase relative to each other ${ }^{22}$.

We now show that we are able to generate entangled photons from a dot with a FSS of over $50 \mu \mathrm{eV}$ at zero field, by simply applying a voltage to the sample. The dot we study has an anticrossing of amplitude $s_{0}=1.5 \mu \mathrm{eV}$ at a field of $-240 \mathrm{kV} \mathrm{cm}^{-1}$. This splitting is below that required to observe entangled-photon emission from the cascade ${ }^{9,10,22}$. To confirm this, polarized crosscorrelation measurements were made between the $X$ and $X_{2}$ transitions at $|s|=s_{0}$ (Fig. $4 \mathrm{a}-\mathrm{c}$ ). The shapes of the peaks are dominated by the Gaussian-like instrument response function with width $\sim 600$ ps. As expected, strong correlation is observed in the rectilinear and diagonal bases (Fig. 4a,b), and strong anticorrelation in the circular basis (Fig. 4c), when the two photons are emitted at closely spaced times. The fidelity of the emission to the Bell state $\Psi^{+}=\left[\left|X^{H} X_{2}{ }^{H}\right\rangle+\left|X^{V} X_{2}{ }^{V}\right\rangle\right] / \sqrt{2}, f^{+}$, is given by $\left[C_{\{\mathrm{H}, \mathrm{V}\}}+C_{\{\mathrm{D}, \mathrm{A}\}}-C_{\{\mathrm{L}, \mathrm{R}\}}+1\right] / 4$ (ref. 22), where $C_{\{\mathrm{H}, \mathrm{V}\}}$ denotes the degree of polarization correlation in the $\{H, V\}$ basis. We obtain 
$f^{+}=71 \pm 3 \%$. Similar measurements with pulsed excitation give $f^{+}=64 \pm 3 \%$ when averaging over all photons emitted within 100 ps of each other (Fig. 4e). At this finite splitting the basis state that has maximum $f^{+}$rotates at a rate proportional to $\left|s_{0}\right|$, which combined with re-excitation causes $f^{+}$to fall to the classical value of 0.25 at times away from zero ${ }^{23}$. In both pulsed and continuous-wave measurements at $s_{0}, f^{+}$is above the threshold of 0.5 , confirming that the emission of entangled photon pairs has not been degraded by the application of such a large electric field. At different values of electric field and increased $|s|$, continuous-wave correlation measurements confirm that as spectral distinguishability is introduced into the cascade $f^{+}$falls as expected (Fig. 4d).

We have demonstrated an effective and versatile technique to control the FSS of a single quantum dot, which has enabled us to observe anticrossings in the fine-structure of single dots. Such electric-field control is well suited to the incorporation of highquality, low-volume cavities facilitating higher efficiencies and cavity quantum electrodynamics effects ${ }^{24,25}$. In future, this technique will allow control of the fine-structure splitting and eigenstates of the exciton on a timescale faster than the radiative lifetime $e^{26,27}$, enabling manipulation of superpositions stored in these states.

\section{Methods}

The InAs dots are grown at the centre of a $10 \mathrm{~nm}$ GaAs quantum well clad with a short-period superlattice equivalent to $\mathrm{Al}_{0.75} \mathrm{Ga}_{0.25}$ As. Doping that extends into the superlattice allows application of an electric field along the growth direction. This $\mathrm{p}-i-\mathrm{n}$ device has a $d=140$-nm-thick $i$-region and is encased in a weak planar microcavity consisting of 14/4 periods below/above the dot layer. Thus, the applied electric field is calculated to be $\left(V-V_{\mathrm{bi}}\right) / d$ where the built-in potential, $V_{\mathrm{bi}}$, is $2.2 \mathrm{~V}$. The $\mathrm{Al}_{0.75} \mathrm{Ga}_{0.25}$ As barrier on either side of the quantum well controls the charging of the dot. Although the dot layer is positioned an equal distance from the $\mathrm{n}$ and $\mathrm{p}$ contact, the tunnelling rates will differ substantially owing to the lower effective mass of the electron and different confinement energies of the carriers.

Excitation and photon collection occurs through an opaque metallic film on the sample surface, patterned with micrometre-diameter apertures (Fig. 1a). During spectroscopy the samples were excited by a continuous-wave $850 \mathrm{~nm}$ laser diode, which creates carriers in the wetting layer and dot. For cross-correlation measurements, a Ti-sapphire laser operating in either the pulsed or continuous-wave regime excited the dots at $850 \mathrm{~nm}$.

Single dots have a characteristic spectral arrangement of optical transitions that can readily be identified as exciton $(X)$, biexciton $\left(X_{2}\right)$ and charged excitons $\left(X^{+}\right.$, $X^{-}$), where this notation refers to the initial state. For the neutral $X_{2}$-to- $X$-to-empty cascade we are able to induce Stark shifts of $25 \mathrm{meV}$ at $500 \mathrm{kV} \mathrm{cm}^{-1}$. A study of several dozen dots emitting in the range $1,310-1,340 \mathrm{meV}$ showed that the FSS at $50 \mathrm{kV} \mathrm{cm}^{-1}$ exhibited a Gaussian distribution centred on $109 \mu \mathrm{eV}$ with width $67 \mu \mathrm{eV}$. Such a distribution fits well with the trend previously reported for dots in GaAs (ref. 28). These observations confirm that placing the dots in a quantum well has not changed their electronic properties.

We determine $|s|=\left|E_{+}-E_{-}\right|$and $\theta$ by measuring spectra polarized at multiple angles $\phi$ to the [110] crystal direction, extracting the energy difference between the exciton and biexciton transitions, $\left(X(\phi)-X_{2}(\phi)\right)$. This technique eliminates small energy shifts induced by rotation of the polarization optics giving measurements of $s(\phi)$ with submicroelectronvolt accuracy ${ }^{1,28}$. When $|s|$ is below the resolution of the system used for this measurement $(\sim 40 \mu \mathrm{eV})$ we observe a sinusoidal variation in $\left(X(\phi)-X_{2}(\phi)\right)$ (Fig. 3e) from which we determine the orientation angle, $\theta$, and magnitude of the FSS, $|s|$, at each field.

Received 1 April 2010; accepted 10 August 2010; published online 3 October 2010

\section{References}

1. Stevenson, R. M. et al. Magnetic field induced reduction in the exciton polarization splitting in InAs quantum dots. Phys. Rev. B 73, 033306 (2006).

2. Jundt, G., Robledo, L., Hogele, A., Falt, S. \& Imamoglu, A. Observation of dressed excitonic states in a single quantum dot. Phys. Rev. Lett. 100, 177401 (2008).

3. Muller, A., Fang, W., Lawall, J. \& Solomon, G. S. Creating polarization-entangled photon pairs from a semiconductor quantum dot using the optical Stark effect. Phys. Rev. Lett. 103, 217402 (2009).
4. Kowalik, K. et al. Monitoring the electrically driven cancellation of exciton fine structure in a semiconductor quantum dot by optical orientation. Appl. Phys. Lett. 91, 183104 (2007).

5. Gerardot, B. D. et al. Manipulating exciton fine structure in quantum dots with a lateral electric field. Appl. Phys. Lett. 90, 041101 (2007).

6. Bayer, M. et al. Electron and hole g factors and exchange interaction from studies of the exciton fine structure in InGaAs quantum dots. Phys. Rev. Lett. 82, 1748-1751 (1999).

7. Gammon, D., Snow, E. S., Shanabrook, B. V., Katzer, D. S. \& Park, D. Homogeneous linewidths in the optical spectrum of a single gallium arsenide quantum dot. Science 273, 87-90 (1996).

8. He, L., Gong, M., Li, C-F., Guo, G-C. \& Zunger, A. Highly reduced fine-structure splitting in InAs/InP quantum dots offering an efficient on-demand entangled 1.55 micrometre photon emitter. Phys. Rev. Lett. 101, 157405 (2008).

9. Stevenson, R. M., Young Cooper, K., Atkinson, P., Ritchie, D. A. \& Shields, A. J. A semiconductor source of entangled photon pairs. Nature 439, 179-182 (2006).

10. Hafenbrak, R. et al. Triggered polarization-entangled photon pairs from a single quantum dot up to $30 \mathrm{~K}$. New. J. Phys. 9, 315 (2007).

11. Singh, R. \& Bester, G. Lower bound for the excitonic fine structure splitting in self assembled quantum dots. Phys. Rev. Lett. 104, 196803 (2010).

12. Seidl, S. et al. Effect of uniaxial stress on excitons in a self-assembled quantum dot. Appl. Phys. Lett. 88, 203113 (2006).

13. Vogel, M. M. et al. Influence of lateral electric fields on multiexcitonic transitions and fine structure of single quantum dots. Appl. Phys. Lett. 91, 051904 (2007).

14. Kowalik, K. et al. Stark spectroscopy of Coulomb interactions in individual InAs/GaAs self-assembled quantum dots. Phys. Status Solidi 3, 3980-3894 (2006).

15. Marcet, S., Ohtani, K. \& Ohno, H. Vertical electric field tuning of the exciton fine structure splitting and photon correlation measurements of GaAs quantum dot. Appl. Phys. Lett. 96, 101117 (2010).

16. Barker, J. A. \& O'Reilly, E. P. Theoretical analysis of electron-hole alignment in InAs-GaAs quantum dots. Phys. Rev. B 61, 13840-13850 (2000).

17. Finley, J. J. et al. Quantum-confined Stark shifts of charged exciton complexes in quantum dots. Phys. Rev. B 70, 201308 (2004).

18. Feynman, R. P., Leighton, R. B. \& Sands, M. The Feynman Lectures on Physics, Vol. 1 (Addison-Weasley, 1963).

19. Khitrova, G., Gibbs, H. M., Kira, H., Koch, S. W. \& Scherer, A. Vacuum Rabi splitting in semiconductors. Nature Phys. 2, 81-90 (2006).

20. Stinaff, E. A. et al. Optical signatures of coupled quantum dots. Science 311, 636-639 (2006)

21. Berthelot, A. et al. Unconventional motional narrowing in the optical spectrum of a semiconductor quantum dot. Nature Phys. 2, 759-764 (2006).

22. Hudson, A. J. et al. Coherence of an entangled exciton-photon state. Phys. Rev. Lett. 99, 266802 (2007).

23. Stevenson, R. M. et al. Evolution of entanglement between distinguishable light states. Phys. Rev. Lett. 101, 171501 (2008).

24. Ellis, D. J. P. et al. Oxide-apertured microcavity single photon emitting diode. Appl. Phys. Lett. 90, 233514 (2007).

25. Böckler, C. et al. Electrically driven high-Q quantum dot-micropillar cavities. Appl. Phys. Lett. 92, 091107 (2008).

26. Bennett, A. J. et al. Electrical control of the uncertainty in the time of single photon emission events. Phys. Rev. B 72, 033316 (2005).

27. McFarlane, J. et al. Gigahertz bandwidth electrical control over a dark exciton based memory bit in a single quantum dot. Appl. Phys. Lett. 94, 093113 (2009).

28. Young, R. J et al. Inversion of exciton level splitting in quantum dots. Phys. Rev. B 72, 113305 (2005).

\section{Acknowledgements}

This work was partly supported by the EU through the Integrated Project QESSENSE and the Marie Curie Framework 7 project Spin-Optronics. EPSRC and TREL provided support for R.B.P., M.P. and A.B.d.I.G. and QIPIRC for C.A.N. We would like to thank D. Granados and R. J. Young for useful discussions on design of the device.

\section{Author contributions}

The samples were grown by I.F., C.A.N. and D.A.R. and processed by R.B.P. The optical measurements were made by A.J.B, M.P. and R.M.S. A.J.S. guided the work. All authors discussed the results and their interpretation. A.J.B wrote the manuscript, with contributions from the other authors.

\section{Additional information}

The authors declare no competing financial interests. Supplementary information accompanies this paper on www.nature.com/naturephysics. Reprints and permissions information is available online at http://npg.nature.com/reprintsandpermissions. Correspondence and requests for materials should be addressed to A.J.B. 